\title{
Observations of Comet P/Shoemaker-Levy 9 Impact on Jupiter from Lick Observatory Using a High Resolution Speckle Imaging Camera
}

\author{
C. $\operatorname{Max}$ \\ D. Gavel \\ E. Johansson \\ B. Sherwood \\ M. Liu \\ B. Bradford
}

RECEIVED

JUN 241990

OST:

March 15, 1996

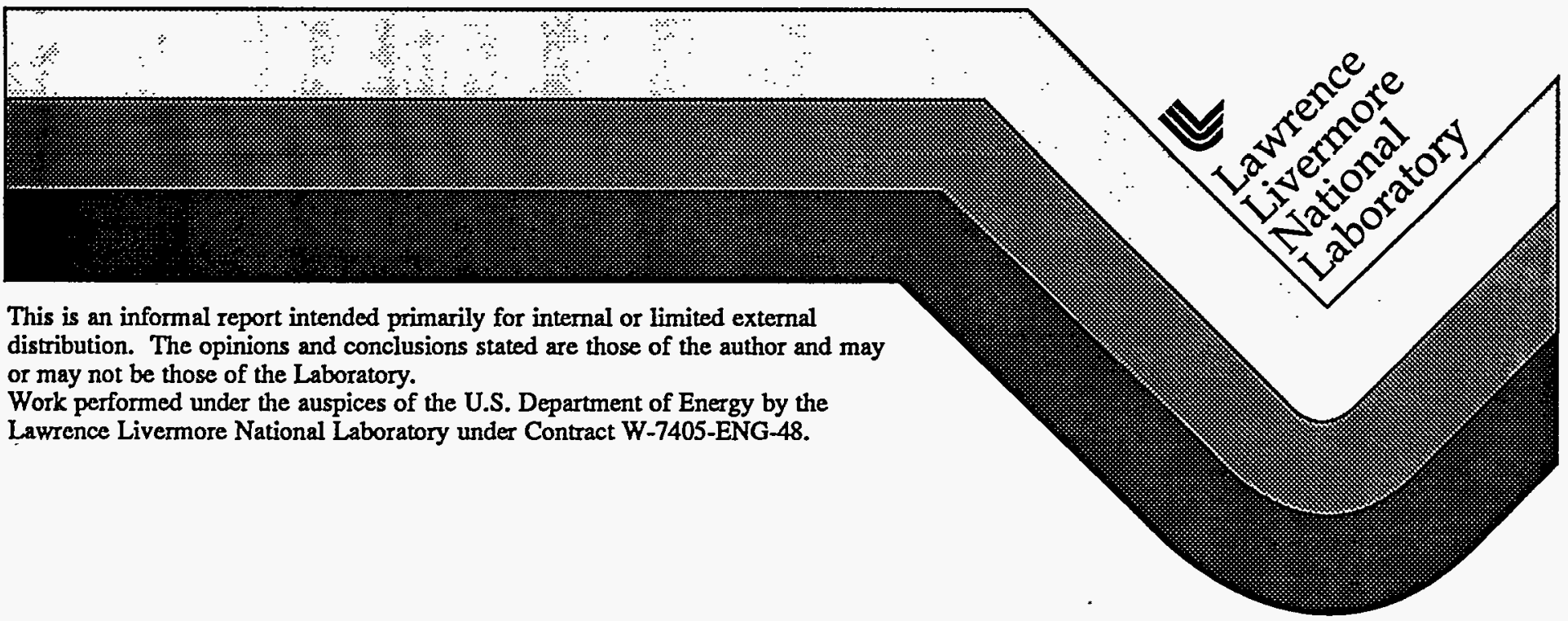




\section{DISCLAIMER}

This document was prepared as an account of work sponsored by an agency of the United States Government. Neither the United States Government nor the University of California nor any of their employees, makes any warranty, express or implied, or assumes any legal liability or responsibility for the accuracy, completeness, or usefulness of any information, apparatus, product, or process disclosed, or represents that its use would not infringe privately owned rights. Reference herein to any specific commercial product, process, or service by trade name, trademark, manufacturer, or otherwise, does not necessarily constitute or imply its endorsement, recommendation, or favoring by the United States Government or the University of California. The views and opinions of authors expressed herein do not necessarily state or reflect those of the United States Government or the University of California, and shall not be used for advertising or product endorsement purposes.

This report has been reproduced directly from the best available copy.

Available to DOE and DOE contractors from the Office of Scientific and Technical Information P.O. Box 62, Oak Ridge, TN 37831

Prices available from (615) 576-8401, FTS 626-8401

Available to the public from the National Technical Information Service

U.S. Department of Commerce 5285 Port Royal Rd., Springfield, VA 22161 


\title{
Observations of Comet P/Shoemaker-Levy 9 Impact on Jupiter from Lick Observatory Using a High Resolution Speckle Imaging Camera
}

\author{
Claire Max, Don Gavel, Erik Johansson, Bob Sherwood \\ Lawrence Livermore National Laboratory \\ Mike Liu \\ University of California, Berkeley \\ Bill Bradford \\ University of California, Santa Cruz
}

\begin{abstract}
During the week of the impacts of Comet Shoemaker-Levy 9 into Jupiter, we used a speckle imaging camera mounted on the Lick Observatory 3 meter Telescope to record a continuous stream of images of the planet. Because the speckle imaging technique compensates for atmospheric blurring, the resulting images were most likely the highest resolution of any taken from the ground. These images compliment the Hubble Space Telescope data by covering time periods when Hubble was not observing Jupiter.

We collected full planet 1024 by 1024 pixel CCD images taken 20 per minute for 4 hours per night over 6 nights July 15 to 22. Only a portion of this raw data has been reduced to high resolution images to date.
\end{abstract}

\section{Observations}

These images were taken at visible and near infrared wavelengths using a high resolution speckle imaging camera on the 3 Meter Telescope.

The speckle imaging technique involves recording several short exposure images and subsequently combining them by computer to yield a single high resolution image $[1,2]$. In a single speckle frame, the exposure is short enough that the turbulent atmosphere is essentially frozen. The resulting focal plane image is a random speckle interference pattern that retains diffraction-limited information about the imaged object. A large number of these image frames are recorded and later processed to form a single high resolution image. The technique overcomes to a great extent the blurring affects of the atmosphere, and in theory can approach the diffraction limit.

For the comet Shoemaker Levy 9 event, we used a large format, high speed readout CCD camera mounted at the Cassegrain focus of the Lick Observatory 3 Meter Telescope $[3,4]$. Earlier planet imaging tests at the 1 meter telescope the previous year, and at the 3 meter in May, showed the feasibility of using speckle imaging to produce high resolution planet images. 
The CCD pixel scale was 0.06 arcseconds per pixel, much smaller than the typical seeing blur size, (about 1 arcsecond on Mt. Hamilton), and about the same size as the diffraction spot size of the 3 meter telescope at a wavelength of 1 micron. The entire disk of Jupiter fits on the $1024 \times 1024 \mathrm{CCD}$ array at this plate scale. The 0.06 arcsecond pixel corresponds to $200 \mathrm{~km}$ on the surface of Jupiter.

We recorded images of Jupiter beginning one day before the first comet fragment impacts, July 15, and continued for the duration of the events, through July 22 . The weather and seeing conditions were generally good, except that one night, July 21, was completely clouded out. With the speckle imaging system, we were able to record one frame every 3 seconds. After each night of observing, the image reconstruction algorithm was run over subsets of data frames to generate representative images for the night. These were then forwarded to the LLNL, JPL, and STSI World-Wide-Web sites for viewing over internet.

Images can be viewed/downloaded from the following internet sites:

http://www.llnl.gov/jupiter/comet.html

http://newproducts.jpl.nasa.gov/sl9/lick.html

$\mathrm{ftp}: / /$ tweety.llnl.gov/pub/jupiter_pictures/new_pictures

\section{Image processing}

The image reconstruction was done using the bispectrum phase algorithm described in the appendix. The resolution achieved on full planet images is about 0.3 to 0.4 arcseconds (features 5 pixels across, corresponding to $1000 \mathrm{~km}$ are discernible). So far, the main limitation to achievable resolution is Jupiter's high rotation rate. Motion blur limits the time span and therefore the number of input speckle frames that can be used to form an output image. Image quality improves with the number of input speckle frames since the speckle reconstruction algorithm relies on a large statistical sample to reduce Poisson and speckle noise. Features at the equator and central meridian appear to move at about 0.2 arcsecond per minute, limiting the number of raw data frames used in the reconstruction, at our 20 frames per minute data collection rate, to 5 for diffractionlimited resolution, 20 for 0.2 arcsecond resolution, etc. We found that the optimum trade-off of frame count with motion blur is about 20 input data frames.

It is possible to increase the number of useful input speckle frames by tracking the planet's rotation. This is accomplished by mapping planet images to a latitude-longitude grid before applying the speckle reconstruction algorithm. This technique is currently under investigation.

\section{Image interpretation}

Figure 1 shows a time sequence of the fragment $G$ impact site. The first frame shows the area as seen on July 17 , before the impact. The next set of four shows the spot as it traversed on its third rotation after impact, as seen on July 19 . The imaging wavelength is $550+/-50 \mathrm{~nm}$. Planet images were reconstructed from 20 speckle data frames then projected to a latitude-longitude grid. The images shown are of intensity, normalized by incident solar flux on an ellipsoid.

The $\mathrm{G}$ impact, typical of large fragment impacts, created a dark inner core and a halo of dark material asymmetrically distributed around the core. The shape of the halo indicates 
that material was ejected above the atmposhere out at an angle, and fell back onto the cloud tops

Comparison of the images shows that the weather patterns surrounding the spot, and even within the ejecta halo, appear the same as before impact, implying that most of the visible dark material is above the cloud layers as opposed to penetrating them. The core appears larger here than in the images taken by Hubble a day earlier. Also, over this sequence there is apparently a slight change of the central core's size with time, but not a similar change in shape of the halo, possibly indicating that the core is being affected by the tropospheric winds over short time periods.

Figure 2 shows the same region in multiple wavelength bands: $550 \mathrm{~nm}, 700 \mathrm{~nm}, 850 \mathrm{~nm}$, and $880 \mathrm{~nm}$ (methane absorption band). The spot is redish in the sense that it appears smaller in the longer wavelength images. The spot appears bright in the methane absorption band, indicating that the material is high in the atmosphere since the reflected sunlight is not absorbed by methane. (The $880+/-30 \mathrm{~nm}$ filter available to us was spectrally broader than the methane absorption band, which is only about $30 \mathrm{~nm}$ wide, hence the planet does not appear as dark as it would in an image taken with a narrower band filter). Figure $3 a$ shows intensity slices through the $G$ spot, normalized by incident solar fTux. Figure $3 \mathrm{~b}$ shows the ratio of (infra)red $(850 \mathrm{~nm})$ to visible $(550 \mathrm{~nm})$ normalized intensity along the same slice, indicating the redishness of the core and halo.

Figure 4 shows the timing of impacts and observations. Dark bars indicate when data was collected with the Lick 3 meter telescope. Grey bars indicate the HST observing schedule. As can be seen, there is very little overlap in the two schedules. The diagonal lines indicate impact site longitude relative to the central meridian (the longitude directly facing earth) as a function of time. Number subscripts on the fragment designations indicate the rotation number of the site since impact.

\section{Appendix. Description of the speckle imaging reconstruction algorithm}

The speckle image $i(x)$ that is obtained from an object $o(x)$ obeys a convolution relationship

$$
i_{n}(x)=p_{n}(x) * o(x)
$$

where $\mathrm{x}$ is position in the field of view and $\mathrm{p}_{\mathrm{n}}(\mathrm{x})$ is the combined atmosphere and telescope point-spread function. The subscript $n$ indicates the speckle frame number. Note that the atmosphere is constantly changing and therefore so is the speckle point spread function. The speckle reconstruction algorithm recovers the object by estimating its Fourier phase and Fourier magnitude components separately. Fourier magnitude is obtained using a standard deconvolution technique that uses the average power spectrum of the speckle frames, $\langle\ln (u)>2$ and a modeled or measured atmosphere-telescope average point-spread function $\left\langle\mathrm{P}_{\mathrm{n}}(\mathrm{u})\right\rangle^{2}$

$$
\left.1 O(u)\right|_{\text {est }}=\left[<\mathrm{I}_{n}(\mathrm{u})>^{2} /<\mathrm{P}_{n}(\mathrm{u})>^{2}\right]^{1 / 2}
$$

where $\mathrm{u}$ is the Fourier domain variable associated with $\mathrm{x}$, and $\langle *\rangle$ indicates averaging over speckle frames. Fourier phase is recovered through processing the bispectrum of the images. The bispectrum of $i_{-} n(x)$ is the complex value

$$
I_{B, n}(u, v)=I_{n}(u) I_{n}(v) I_{n}(-u-v)
$$


where $u$ and $v$ are Fourier domain variables and $I \_n(u)$ is the Fourier transform of $i_{-} n(x)$. The bispectrum phase of the object is recovered according to

$$
\arg \left\{\mathrm{OB}_{\mathrm{B}}(\mathrm{u}, \mathrm{v})\right\}=\arg \left\{<\mathrm{IB}_{\mathrm{B}} \mathrm{n}(\mathrm{u}, \mathrm{v})>\right\}
$$

where $<I \_B, n(u, v)>$ is the average bispectrum of the speckle frames. Fourier phase is recovered directly from bispectrum phase by a simple recursion relation. The key element in speckle image processing is the fact that the bispectrum phase of the aberration point-spread function is zero, and thus phase information about the object is recoverable.

There are two main error sources in bispectrum approach to image reconstruction. One is the assumption that the point spread function is constant over the entire object field (the .isoplanatic wavefront assumption). For a large object like Jupiter, 40 arcseconds across, the atmospheric point spread varies to a great extent and so this assumption is violated. Second, the algorithm depends on statistical averaging over many speckle frames. A limited number of available frames will increase the noise in the estimates. This noise most severely affects the high spatial frequency components of the bispectrum and magnitude estimates and therefore limits reconstructed image resolution.

\section{References}

1. Labeyrie, A. 1970, Attainment of diffraction limited resolution in large telescopes by Fourier analysing speckle patterns in star images, Astron. Astrophys, 6, 85-87.

2. Lawrence, T. W., D. M. Goodman, E. M. Johansson, \& J. P. Fitch, 1992, Speckle imaging of satellites at the U.S. Air Force Maui Optical Station, Applied Optics, 31, (29), 6307-6321.

3. Max, C. E., Presentation at the AAS Division of Planetary Sciences Conference, Washington, DC, 1994.

4. Gavel, D. T. and E. M. Johansson, Poster presentation at the Comet Day Symposium, High Velocity Impact Symposium, Santa Fe, NM, October, 1994. 


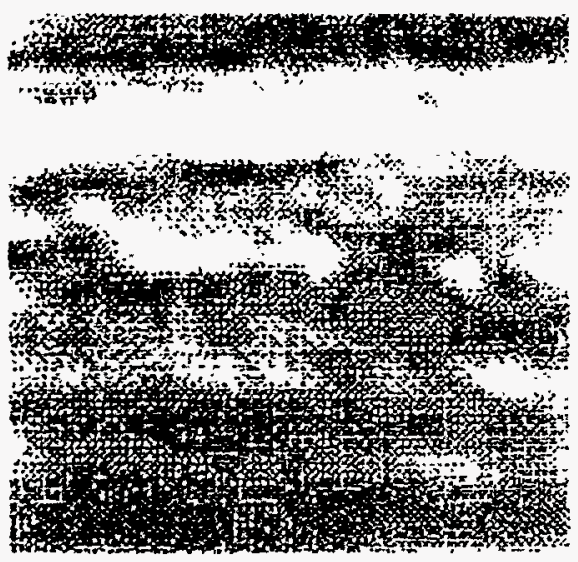

July $17,4: 46$ UT

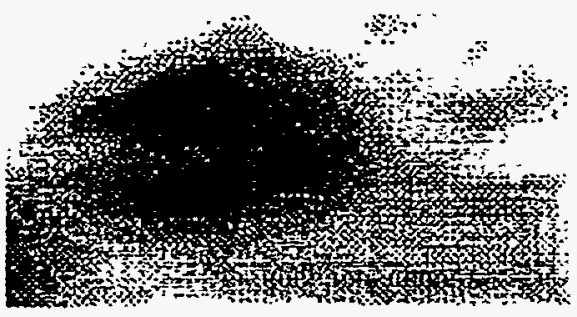

July $19,3: 32$ UT

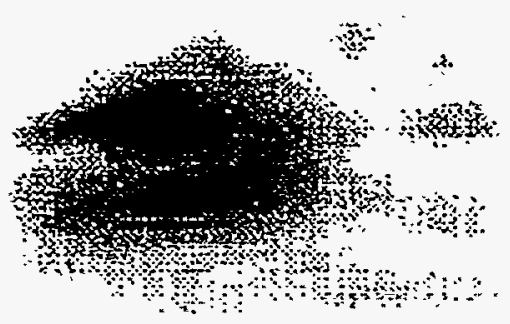

July 19, 3:50 UT

H.

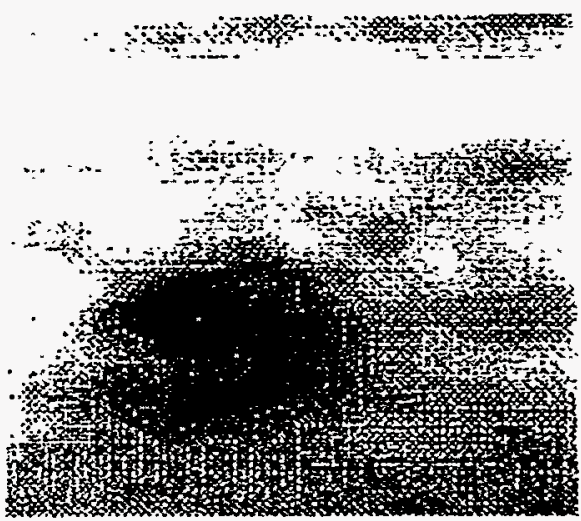

July $19,4: 46$ UT

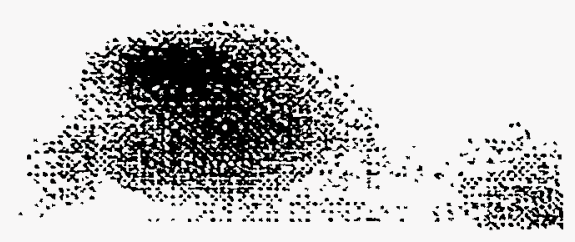

July $19,6: 20$ UT

July 19, 4:24 UT

Figure 1. Time sequence of images of the $G$ impact region 


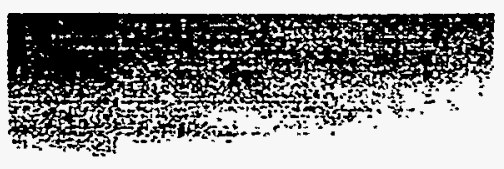

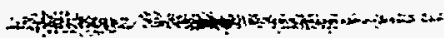
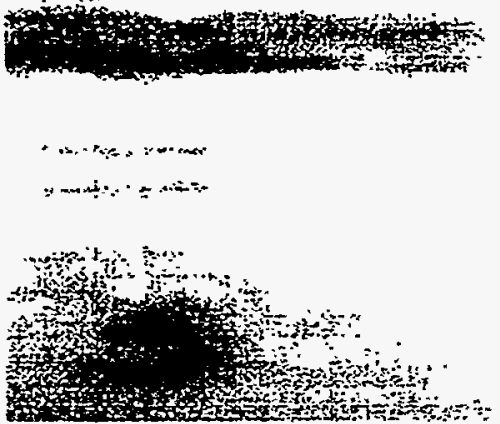

$550 \mathrm{~nm}$

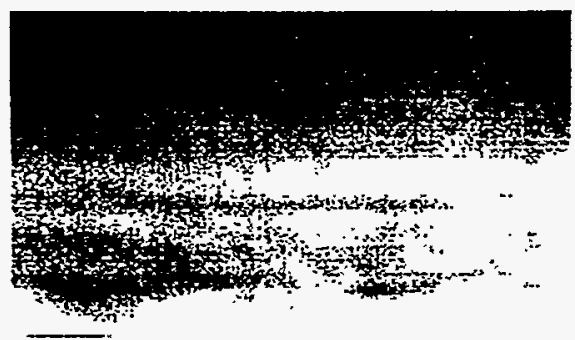

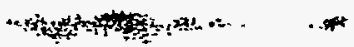

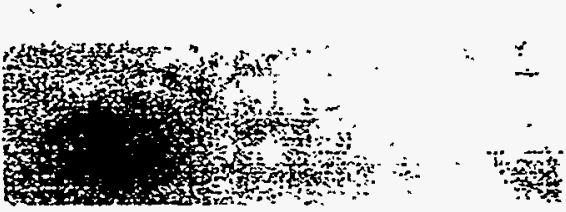

$850 \mathrm{~nm}$

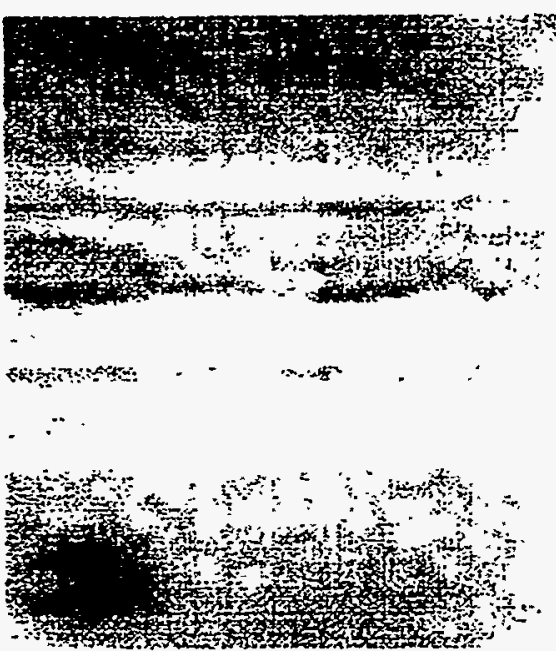

$700 \mathrm{~nm}$

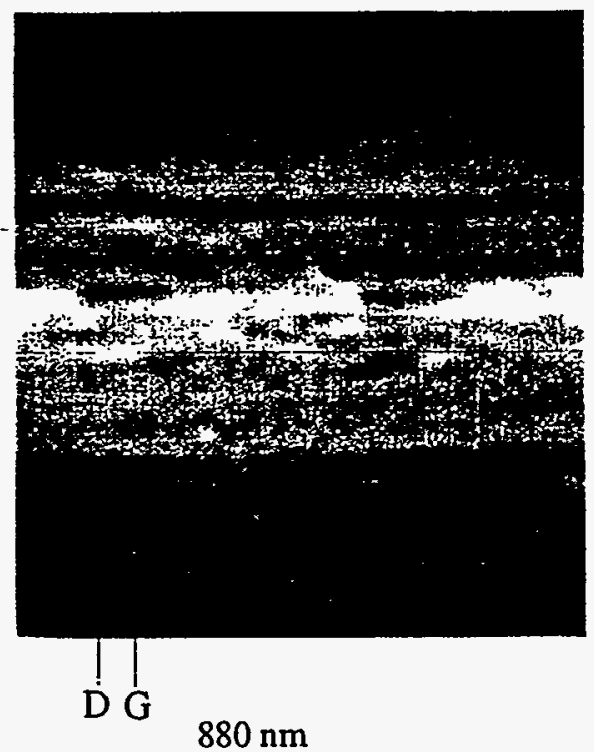

Figure 2. $G$ impact site imaged at various wavelengths 


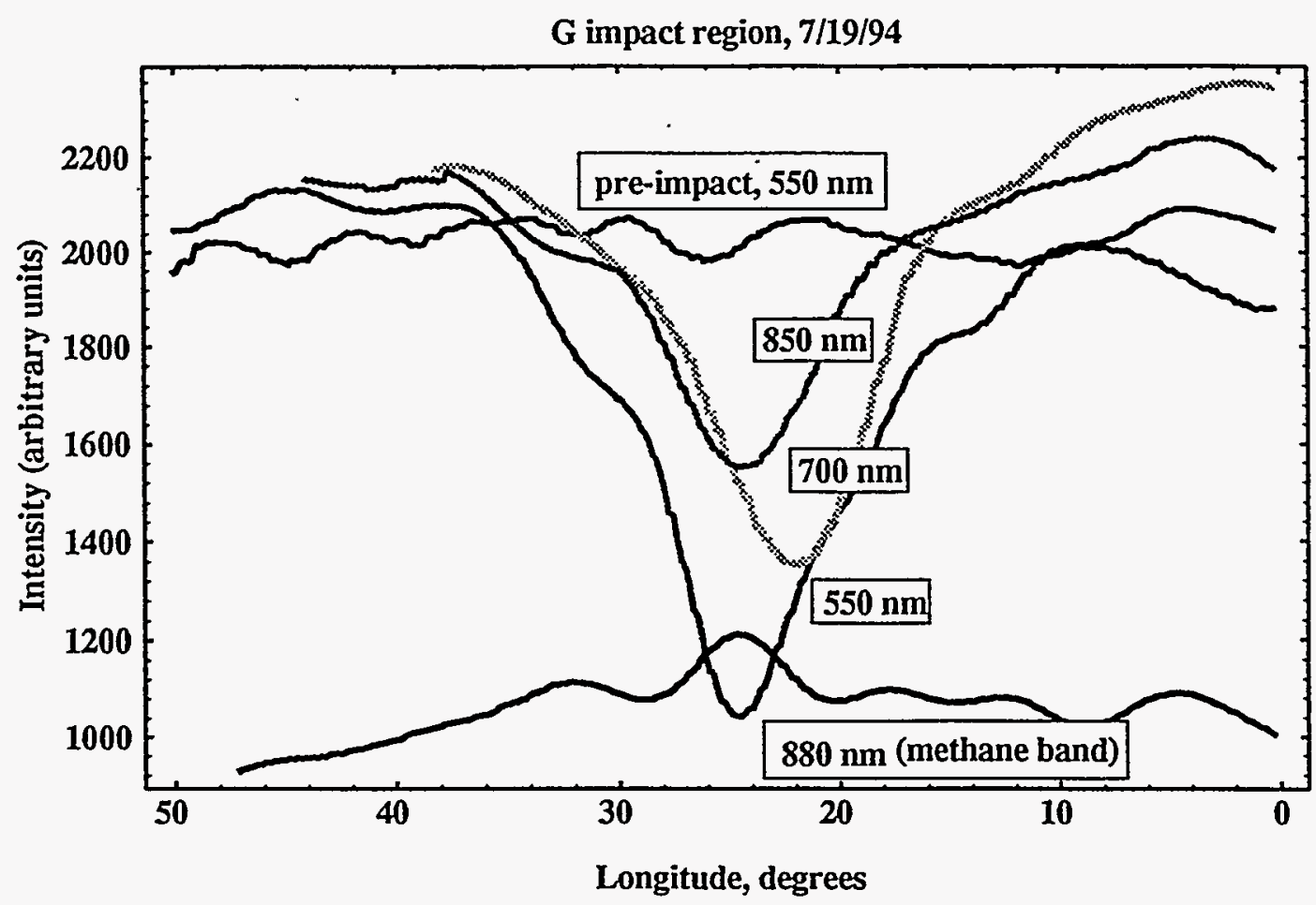

Figure 3a. Intensity slices through the $\mathrm{G}$ impact region at various wavelengths (normalized to solar illumination function)

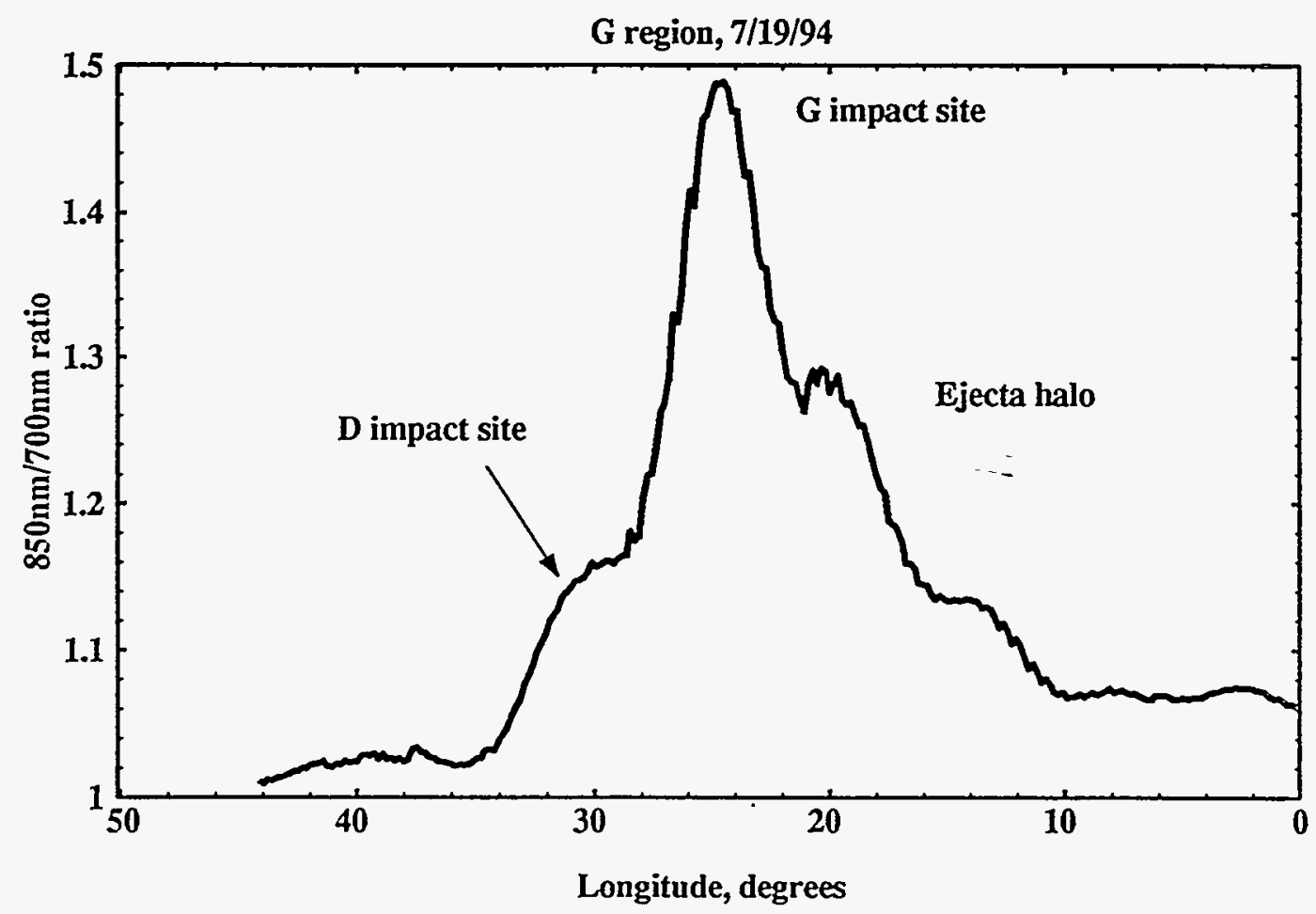

Figure 3b. Ratio of infrared $(850 \mathrm{~nm})$ to visible $(550 \mathrm{~nm})$ intensities in the $\mathrm{G}$ impact region 


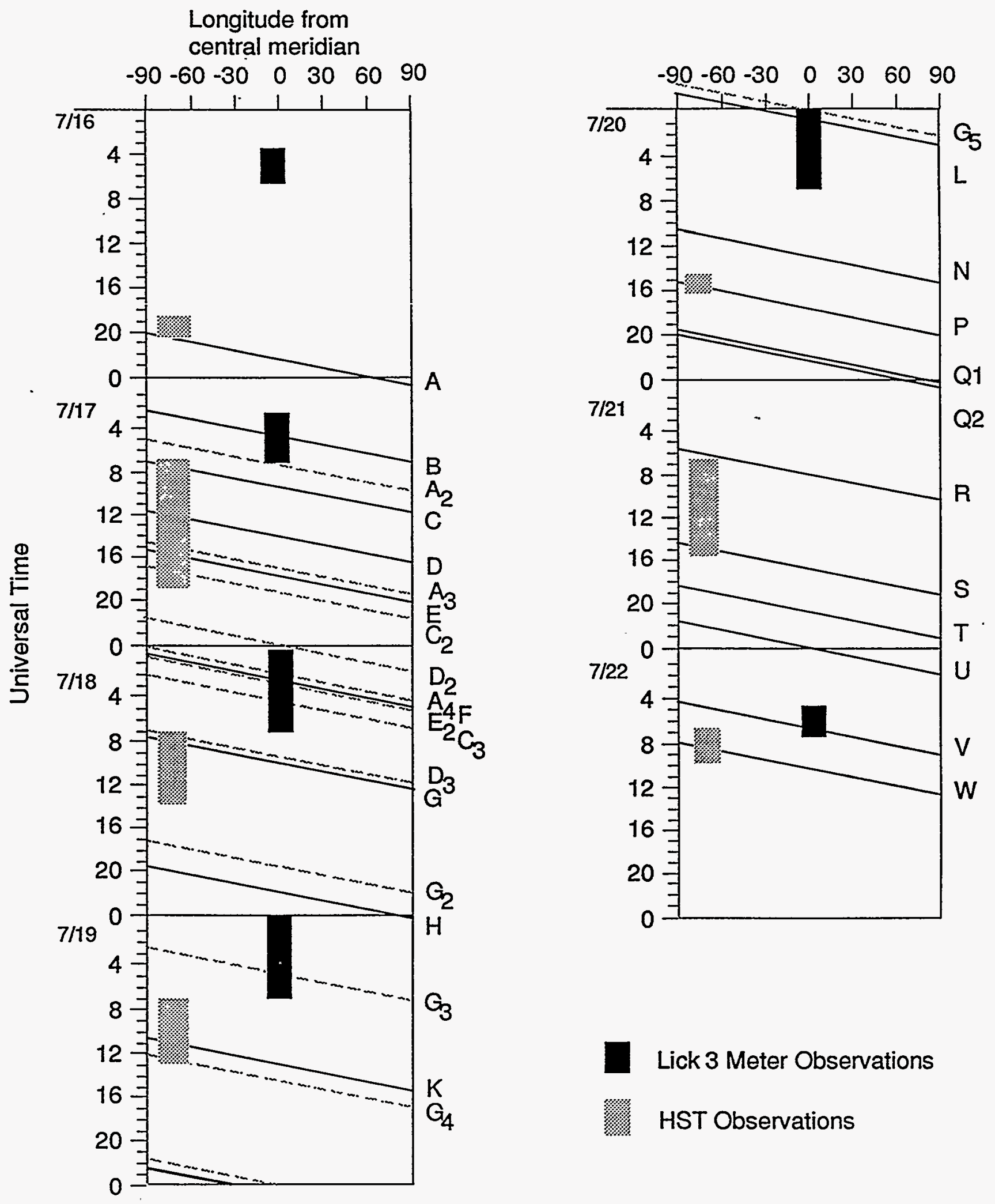

Figure 4. Timing of impacts and observations. Comet fragments are designated by the letters A-W. Numerical subscripts are the age of the impact site in Jupiter rotations. 


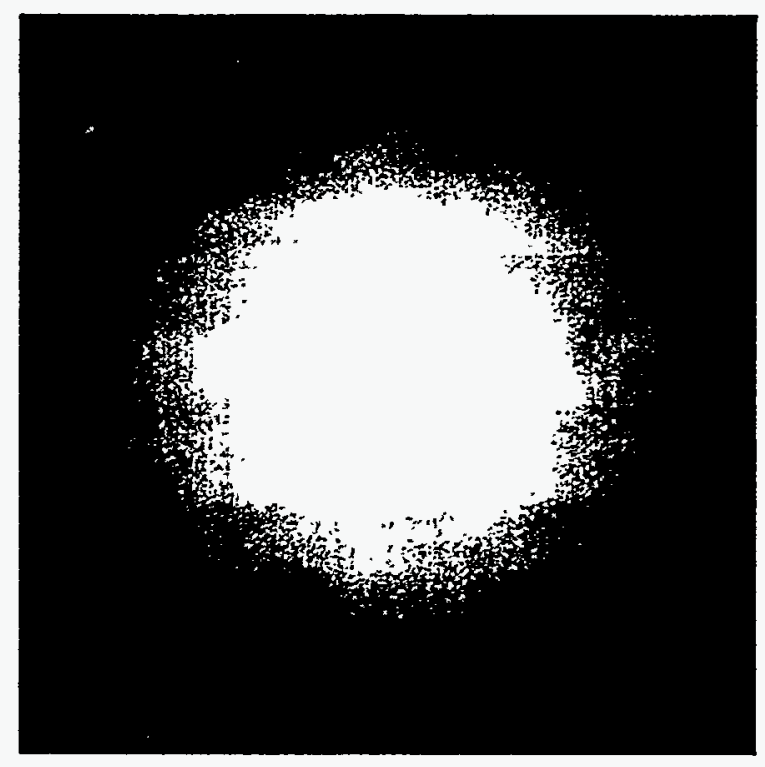

L impact region July 20 00:20 UT

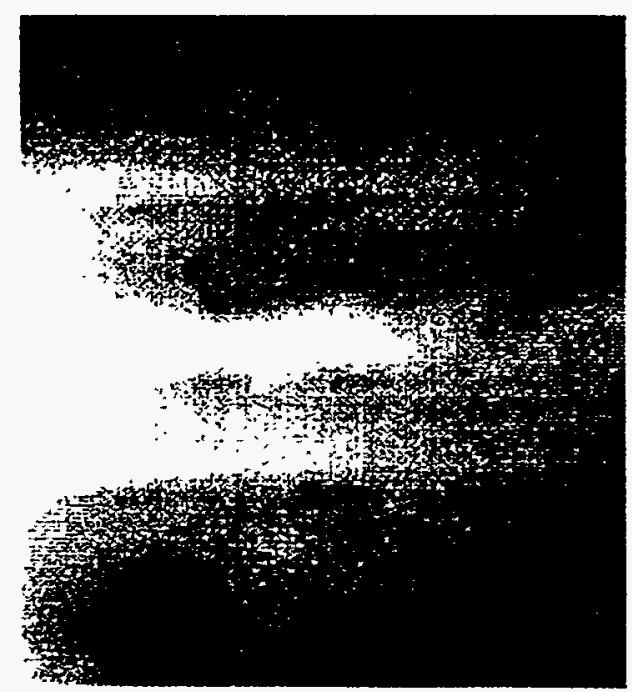

Latitude-longitude map

These images were taken two hours after the impact of fragment $L$. This was 5:20 local time ( 2 hours before sunset). The filter is $850 \mathrm{~nm}$. 


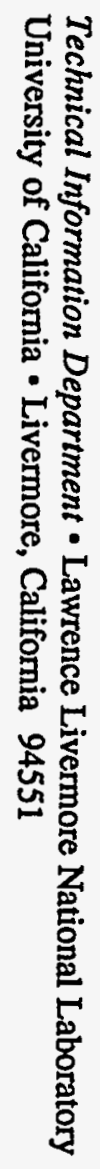

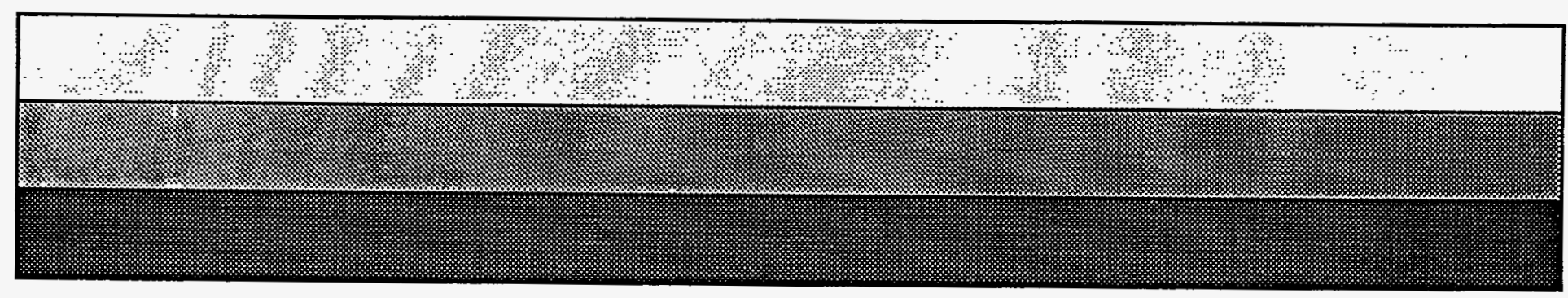

83 Recycled 